\title{
Type la supernovae and the formation of single low-mass white dwarfs
}

\author{
S. Justham ${ }^{1, \star}$, C. Wolf ${ }^{1}, \mathrm{Ph}$. Podsiadlowski ${ }^{1}$, and Zh. Han ${ }^{2}$ \\ 1 Department of Physics, Oxford University, Keble Road, Oxford, OX1 3RH, UK \\ e-mail: sjustham@astro.ox.ac.uk \\ 2 National Astronomical Observatories/Yunnan Observatory, Chinese Academy of Sciences, Kunming, 650011, PR China
}

Received 1 May 2008 / Accepted 13 November 2008

\begin{abstract}
Context. There is still considerable debate over the progenitors of type Ia supernovae (SNe Ia). Likewise, it is not agreed how single white dwarfs with masses $\lesssim 0.5 M_{\odot}$ can be formed in the field, even though they are known to exist.

Aims. We consider whether single low-mass white dwarfs (LMWDs) could have been formed in binary systems where their companions have exploded as an SN Ia. In this model, the observed single LMWDs are the remnants of giant-branch donor stars whose envelopes have been stripped off by the supernova explosion.

Methods. We investigate the likely remnants of SNe Ia, including the effects of the explosion on the envelope of the donor star. We also use evolutionary arguments to examine alternative formation channels for single LMWDs. In addition, we calculate the expected kinematics of the potential remnants of SNe Ia.

Results. SN Ia in systems with giant-branch donor stars can naturally explain the production of single LMWDs. It seems difficult for any other formation mechanism to account for the observations, especially for those single LMWDs with masses $\lesssim 0.4 M_{\odot}$. Independent of those results, we find that the kinematics of one potentially useful population containing single LMWDs is consistent with our model. Studying remnant white-dwarf kinematics seems to be a promising way to investigate SN Ia progenitors.

Conclusions. The existence of single LMWDs appears to constitute evidence for the production of SNe Ia in binary systems with a red-giant donor star. Other single white dwarfs with higher space velocities support a second, probably dominant, population of SN Ia progenitors which contained main-sequence or subgiant donor stars at the time of explosion. The runaway stars LP 400-22 and US 708 suggest the possibility of a third formation channnel for some SNe Ia in systems where the donor stars are hot subdwarfs.
\end{abstract}

Key words. stars: binaries: close - stars: supernovae: general - stars: white dwarfs - stars: kinematics

\section{Introduction}

Type Ia supernovae (SNe Ia) are of major astrophysical importance. They have acquired particular cosmological significance since they have been used to measure the expansion history of the Universe (Riess et al. 1998; Perlmutter et al. 1999; Riess et al. 2004). Understanding their nature is also of importance for understanding the metallicity evolution and star-formation history of galaxies (e.g. Canal et al. 1996; Matteucci \& Recchi 2001). Despite their importance, there is still no agreement on the nature of their progenitors.

There is broad agreement that the destruction of a white dwarf (WD) in a thermonuclear explosion constitutes the supernova event itself, but there are two main classes of competing models for the events which lead to the explosion. In the single-degenerate scenario, the doomed WD accretes matter from a non-degenerate companion (Whelan \& Iben 1973; Nomoto 1982; Han \& Podsiadlowski 2004). In the doubledegenerate scenario, the mass donor is a second WD; the most commonly considered scenario involves the merger of two CO WDs (Iben \& Tutukov 1984; Webbink 1984; also see Martin et al. 2006, for a variant of this scenario). An explosion following the merger of two WDs would leave no remnant, whilst the

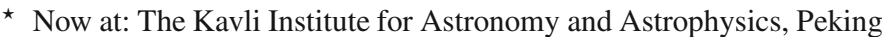
University, Beijing, PR China e-mail: sjustham@kiaa.pku.edu.cn companion star in the single-degenerate scenario would survive and be potentially identifiable (Ruiz-Lapuente 1997; Podsiadlowski 2003; Ruiz-Lapuente et al. 2004).

There has been no conclusive proof to date that any individual object is the surviving non-degenerate donor from an SN Ia explosion. If Chandrasekhar mass WD-WD mergers do not lead to $\mathrm{SNe}$ Ia, they are expected to leave a remnant neutron star via acretion-induced collapse (AIC, see Nomoto \& Iben 1985; Nomoto \& Kondo 1991; but see also Yoon et al. 2007). At present we do not know whether all WD-WD mergers do leave remnants - in which case the double degenerate scenario could not be responsible for $\mathrm{SNe} \mathrm{Ia}$ - and it seems unlikely that that this will become clear in the near future (but see, e.g. Levan et al. 2006). Hansen (2003) first noticed that observed high-velocity WDs (Oppenheimer et al. 2001) could have been produced through SNe Ia; such WDs would be the descendants of non-degenerate mass donors in the pre-supernova binaries. Hansen's idea seems to be consistent with more detailed work on the ages of the WDs in the Oppenheimer et al. sample by Bergeron et al. (2005) and deserves further attention, but by itself it is not a clinching argument for the single-degenerate channel. Nor has the evidence that the SN Ia rate is different for different stellar populations (Mannucci et al. 2005) led to firm conclusions. The strongest direct evidence that non-degenerate donor stars can lead to normal type Ia supernovae has been 
provided by Patat et al. (2007), who observed circumstellar material around SN 2006X which seems extremely hard to reconcile with a double-degenerate progenitor.

Here we suggest that the observed, apparently single, lowmass white dwarfs (LMWDs) provide evidence that at least some SN Ia explosions have occurred with non-degenerate donor stars. We define LMWDs as WDs which are too low in mass to have been produced by single-star evolution as we currently understand it. A population of single LMWDs has been implied by, e.g. the work of Maxted et al. (2000a) ${ }^{1}$. We also investigate the apparently single ultra-cool white dwarfs (UCWDs) as potentially containing a useful subset of the LMWD population and indicate how further observations of the kinematics of this and other populations could lead to constraints on the progenitors of SNe Ia.

In Sects. 2 and 3 we argue that the existence of single LMWDs is most naturally explained by the singledegenerate model for SNe Ia. In Sect. 4 we introduce UCWDs, discuss to what extent the observed single UCWDs might be a useful sample of LMWDs, and consider in what way the observed single UCWD population is consistent with an SN Ia origin.

\section{Formation channels for single LMWDs}

Current evidence suggests that $1 M_{\odot}$ zero-age main-sequence (ZAMS) stars, left to evolve in isolation, produce white dwarfs $\gtrsim 0.55 M_{\odot}$ (e.g. Han et al. 1994; Weidemann 2000). In order to produce low-mass helium WDs (with masses $\lesssim 0.5 M_{\odot}$ ), it is necessary to remove the envelope of a star before it is able to ignite helium. Within the age of the Universe, it is almost certainly impossible for a single star to produce WDs with masses close to $0.3 M_{\odot}$, or even $0.17 M_{\odot}$, as recently inferred for the runaway WD LP 400-22 (Kawka et al. 2006), or $0.23 M_{\odot}$, the estimated mass of the apparently single UCWD LHS 3250 (Bergeron \& Legget 2002).

\subsection{Production of single LMWDs by single stars?}

It would be simplistic to conclude from the existence of currently single LMWDs that single stars can produce LMWDs. However, arguments have been made in favour of a single star channel for the production of some LMWDs, at least in significantly metalrich populations (Kalirai et al. 2007; Kilic et al. 2007c; see also Han et al. 1994). Whilst this is a possibility, that interpretation is not the only one which can explain the observations: this paper provides one alternative applicable to both field stars and clusters, and dynamical interactions in dense clusters may provide another route (e.g. Adams et al. 2004) ${ }^{2}$. Bedin et al. (2008) and Van Loon et al. (2008) do not support the single-star formation channel for LMWDs suggested by Kalirai et al. $(2007)^{3}$.

Even if some WDs less massive than $\sim 0.55 M_{\odot}$ can be produced by single-star evolution with super-solar metallicity, the masses are unlikely to approach those of LP 400-22 or

\footnotetext{
${ }^{1}$ See also Marsh et al. (1995); their study was originally motivated by their interest in finding double degenerate binaries as potentional SN Ia progenitors.

2 Note that mass segregation tends to move low-mass objects outwards in such clusters, so LMWDs might be formed in the core and observed far from the centre.

${ }^{3}$ Bedin et al. argue that there may not, after all, be an unusual single LMWD population in NGC 6791 and Van Loon et al. see no evidence for enhanced stellar mass loss in infrared observations of the cluster.
}

LHS 3250 (as above). Follow-up calculations to Han et al. (1994) imply that only LMWDs with $M \gtrsim 0.4 M_{\odot}$ might be produced from such a single-star channel, even at high metallicity (Meng et al. 2008). Kilic et al. (2007c) also state that their proposed single star channel is highly unlikely to be relevant for WD masses as low as $0.2 M_{\odot}$.

Nothing of what follows would be significantly affected if the maximum mass of the LMWD category had to be revised downwards slightly to account for non-standard single-star evolution. We will mostly be considering LMWDs with masses $\lesssim 0.3 M_{\odot}$, at which point any presently proposed single-star channel is not expected to contribute.

\subsection{Production of single LMWDs by binary stars?}

In binary systems we can invoke mass transfer (and sometimes ablation by a pulsar companion) in order to explain the observed binary LMWDs (e.g. van Kerkwijk et al. 2000; Liebert et al. 2004). Apparently single LMWDs must also be formed within an interacting binary system - either we have not detected their companion or the binary has been disrupted. One attractive formation channel stands out: the formation of the LMWD in a binary where the binary companion exploded in an SN Ia. Before examining this channel in more detail, we discuss possible alternative explanations for single LMWDs.

\subsubsection{Alternatives to $\mathrm{SN}$ la: Core-collapse supernovae?}

The natural alternative to an SN Ia in explaining the disruption of a binary is a core-collapse supernova (chosen such that the system becomes unbound). However, forming an LMWD in such a system is challenging. The binary must remain intact long enough for the WD progenitor to lose its envelope such that it will later form an LMWD; this implies that the initially more massive star has transferred its envelope to the secondary and then becomes a WD. In order to form an LMWD, the primary mass must be $\leqslant 4.5 M_{\odot}$, which our own stellar calculations find to have a core mass at the end of the main sequence of $\approx 0.52 M_{\odot} \cdot{ }^{4}$ In order to produce a core-collapse supernova (requiring $M_{\text {ZAMS }} \gtrsim 8 M_{\odot}$ ), the initially less massive star would thus have to accrete the large majority of the envelope of the primary soon after the primary has left the main sequence, and the initial mass ratio would have to be close to 1 . This highly optimistic scenario does not produce a distinctly low-mass WD; to produce a $0.3 M_{\odot}$ WD in this way requires a ZAMS mass for the primary of $\approx 3 M_{\odot}$, precluding a core-collapse supernova in the binary system.

Our arguments above are generous; Davies et al. (2002) found a much more restrictive result. In a different context they investigated the evolution of systems where a WD is formed before a core-collapse supernova occurs in the system. They concluded that "... the mass of the white dwarfs generated in this way, $M_{\mathrm{WD}} \gtrsim 1 M_{\odot}$ ".

\subsubsection{Alternatives to $\mathrm{SN}$ la: Circumbinary discs?}

If cataclysmic-variable (CV) evolution is driven by circumbinary discs, the donor star may eventually be entirely consumed (the "White Widow" scenario; see Spruit \& Taam 2003, following Spruit \& Taam 2001; Taam \& Spruit 2001). For this mechanism

\footnotetext{
4 We use Eggleton's stellar evolution code (Eggleton 1971; Pols et al. 1995 ) with a metallicity of 0.02 along with the convective overshooting calibration of Pols et al. (1998).
} 
to explain single LMWDs, the WD in the progenitor CV must also have been an LMWD; the WD may not gain much mass, as the matter it accretes can be ejected via nova explosions, but it is unlikely to become significantly less massive.

It is not clear whether this mechanism operates in CVs: attempts to detect circumbinary discs have inferred disc masses several orders of magnitude below the required values (Dubus et al. 2004; Muno \& Mauerhan 2006). Hence in the abscence of further supporting evidence we consider this potential formation channel unlikely at present.

\subsubsection{Alternatives to $\mathrm{SN}$ la: accretion-induced collapse?}

Some systems will contain an accreting WD which succeeds in reaching the Chandrasekhar mass but fails to produce a supernova as the WD is predominantly composed of oxygen, neon \& magnesium $(\mathrm{ONeMg})$ rather than carbon and oxygen $(\mathrm{CO})$. This can occur either because the WD began accreting as an $\mathrm{ONeMg}$ WD or because the accretion rate onto the WD did not allow the WD to remain a CO WD (e.g. Nomoto \& Iben 1985; Nomoto \& Kondo 1991; Martin et al. 2006). Such WDs will produce a neutron star (NS) via AIC. Currently it does not appear likely that AIC produces sufficiently large kicks to disrupt such close binaries (see, e.g. Podsiadlowski et al. 2004).

\subsubsection{Alternatives to SN la: white-dwarf mergers?}

Single LMWDs may be the product of the merger of two lowmass He WDs, with formation rates comparable to or greater than the SN Ia rate (see, e.g. Han et al. 2002, and references therein). However, Han et al. predict masses in excess of $0.4 M_{\odot}$.

\section{Single-degenerate SN la populations}

\subsection{Expected formation channels}

We do not present an exhaustive description of the full evolutionary histories for single-denegerate SN Ia progenitors (see, e.g. Whelan \& Iben 1973; Nomoto 1982; van den Heuvel et al. 1992; Rappaport et al. 1994; Hachisu et al. 1996, 1999; Li \& van den Heuvel 1997; Langer et al. 2000; Hachisu \& Kato 2001; Han \& Podsiadlowski 2004). There is no clear consensus on which donor stars are likely to produce a type Ia supernova. The favoured options involve either donors on the main sequence (MS) or the subgiant branch (known as the supersoft channel), or red-giant (RG) donors.

While the supersoft channel (e.g. Han \& Podsiadlowski 2004) is arguably the favoured channel for the majority of SNe Ia, Hachisu et al. (1996, 1996) and Hachisu \& Kato (2001) suggest situations in which a low-mass giant star may take a WD to the Chandrasekhar mass $M_{\mathrm{Ch}}$ at long orbital periods ${ }^{5}$. Sokoloski et al. (2006) used the 2006 outburst of RS Ophiuchi to confirm the conclusions of Hachisu \& Kato by inferring that RS Oph contains a very massive WD $\left(M_{\mathrm{WD}} \approx 1.4 M_{\odot}\right)$. It is worth noting that we cannot be sure that RS Oph contains a $\mathrm{CO}$ WD rather than an ONeMg one and so we cannot be sure that it will explode rather than collapse. Observational support for a giant donor in a system which produced an SN Ia has been provided via the observations by Patat et al. (2007) of SN 2006X.

\footnotetext{
5 The point at which an explosive nuclear runaway occurs in a nonrotating CO WD is slightly below the Chandrasekhar mass: Nomoto et al. (1984) calculated a mass of $\sim 1.378 M_{\odot}$.
}

King et al. (2003) have also suggested that an accreting WD may not reach $M_{\mathrm{Ch}}$ via the supersoft channel alone, but that a later phase of WD growth could occur in long-period dwarf novae. They argue that, even though the average mass-transfer rate does not reach the steady-burning band (Paczyński \& Żytkow 1978; Nomoto \& Kondo 1991), the accretion rate may be high enough for the WD to grow during dwarf nova outbursts driven by the thermal-viscous disc instability (Cannizzo et al. 1982). Providing the correct mass-accretion rate for the $\mathrm{CO} W D$ to grow to $M_{\mathrm{Ch}}$ is a significant uncertainty in all these models.

\subsection{Remnant mass}

In order to understand the formation of LMWDs in systems which produce SN Ia explosions, we must consider the mass and evolutionary stage of the donor star at the point of the explosion and also the extent to which the donor loses mass because of the explosion. There is a clear division between pre-giant and giant donor stars, with giant donors apparently able to leave LMWD remnants.

Marietta et al. (2000) performed numerical simulations of the effect of an SN Ia explosion on the companion star. They found that 0.15 to $0.17 M_{\odot}$ is stripped away from a $1 M_{\odot}$ mainsequence or subgiant companion by the high-velocity ejecta. Han \& Podsiadlowski (2004) found in their population synthesis simulations of the supersoft channel that, at the time of the explosion, the companion has a mass between $\gtrsim 0.5 M_{\odot}$ and $2.2 M_{\odot}$, with a typical mass of $1 M_{\odot}$ (for more details see also Han 2008). Applying the results of Marietta et al. as a percentage $-15 \%$ of the donor mass - leads to a lowest estimated remnant mass of $\approx 0.42 M_{\odot}$. If the WD explodes as it reaches $M_{\mathrm{Ch}}$, then this remant mass is a lower limit for the MS channel, assuming negligible subsequent mass loss in a wind. Hachisu \& Kato (2001) found a lower limit on the mass of the donor from the supersoft channel (at the time of the $\mathrm{SN}$ ) of $>1.3 M_{\odot}$ (assuming an initial white dwarf mass of $1 M_{\odot}$ ). Despite these differences, both studies suggest that it is difficult to produce LMWDs via main-sequence or subgiant donors.

Marietta et al. also found that a red-giant donor will lose almost its entire envelope (96\%-98\%) due to the impact of the SN Ia explosion and leave only the core of the star, providing a possible pathway for the formation of a subset of single, lowmass He WDs ${ }^{6}$. For the RG channel, Hachisu \& Kato (2001) found a lower limit on the total donor mass of $\gtrsim 0.4 M_{\odot}$. If the RG channel produces SNe Ia, then ram-pressure stripping of the donor's envelope would be expected to lead to the formation of LMWDs. The remnant WD mass is dependent on the core mass of the donor at explosion and is therefore strongly correlated with the orbital period (see Sect. 3.4).

One formation channel that is rarely discussed in the literature is one where the donor star is a hot subdwarf star (see, e.g. Geier et al. 2007). We do not expect significant stripping of the donor by the supernova ejecta in this case, as the donor star will be tightly bound, but the mass of the donor star could easily be low enough for a single LMWD to be formed by the natural evolution of the donor star.

\subsection{Sub-Chandrasekhar mass explosions}

A variation on the above models for $\mathrm{SNe}$ Ia involves the explosion of sub- $M_{\mathrm{Ch}} \mathrm{CO}$ WDs covered with a thick helium layer (Woosley \& Weaver 1994). In that model, the detonation of the

\footnotetext{
${ }^{6}$ See also Iben \& Tutukov (1984).
} 
helium layer is responsible for triggering the supernova. Fink et al. (2007) found that sub- $M_{\mathrm{Ch}}$ explosions were unlikely to be able to explain either normal or subluminous $\mathrm{SNe} I a$, but there could be implications for our LMWD formation channel if a significant fraction of SNe Ia were found to be produced by sub$M_{\mathrm{Ch}}$ detonations ${ }^{7}$. Qualitatively there would be little change to our model, as the RG donors would still be stripped of their envelopes and produce LMWDs. The quantitative remnant mass distribution may be different. For example, these sub- $M_{\mathrm{Ch}}$ explosions might plausibly happen when the donor stars are lower on the giant branch than for the standard model. In that case, the typical remnant WDs may be less massive, the orbital periods at explosion lower and the runaway remnant velocities higher than for detonations at $M_{\mathrm{Ch}}$. The remnant velocity is considered hereafter as a diagnostic of the orbital period at explosion, assuming Chandrasekhar-mass explosions.

\subsection{Binary orbits and runaway velocities}

If single LMWDs have been released from binary systems in which the other component has exploded as a type Ia supernova, the space velocity of the remnant should be a useful diagnostic of the orbital period at explosion. As our arguments above suggest that the LMWDs are most likely to originate in systems with redgiant donor stars, the relationship between core mass and orbital period in such systems can act as a further constraint.

In what follows we assume that the donor stars are filling their Roche lobes, as it seems to us that the mass transfer in systems which produce an SN Ia is most likely to be due to Roche-lobe overflow. However, it is not known whether this is the case as, for example, it is unclear whether the donor star in RS Ophiuchi is rotating synchronously with the binary orbit (see, e.g. Murset \& Schmid 1999; Zamanov et al. 2007). If the donor stars do not fill their Roche lobes, then the following method may slightly overestimate the runaway velocities for a given remnant mass.

If we define $q$ as $M_{2} / M_{1}$ (where $M_{1}$ is the mass of the SN Ia progenitor and $M_{2}$ is the mass of the companion producing the LMWD), write the total mass of the system (in solar units) as $M_{\text {tot, } \odot}$ and the pre-SN orbital period (in days) as $P_{\text {days }}$, we can write the pre-SN orbital velocity as:

$V_{\text {orb }} \approx \frac{213}{1+q}\left(\frac{M_{\text {tot, } \odot}}{P_{\text {days }}}\right)^{1 / 3} \mathrm{~km} \mathrm{~s}^{-1}$.

This shows that, for $M_{2} \ll M_{1}$, the orbital velocity is relatively insensitive to the donor mass.

Furthermore, assuming that the remnant WD mass equates to the core mass of the donor at the time of the SN explosion, then the well-defined relationship between core mass and radius for giant-branch donors leads to an expression for the orbital period of the system at the supernova stage. Rappaport et al. (1995) found the period-mass relation:

$P_{\text {orb }} \approx 0.374\left(\frac{R_{0} M_{\mathrm{wd}}^{4.5}}{1+4 M_{\mathrm{wd}}^{4}}+0.5\right)^{3 / 2} M_{\mathrm{wd}}^{-1 / 2}$ days,

where $M_{\mathrm{wd}}$ is the mass of the future WD (currently the core of the giant star) in units of solar masses. Their preferred value for the fitting parameter $R_{0}$ was $4950 R_{\odot}$, which we also adopt. If

\footnotetext{
7 If such sub- $M_{\mathrm{Ch}}$ explosions occur but are not seen as $\mathrm{SN}$ Ia, it is not clear to us whether such an event would remove the RG donor's envelope in order to produce a LMWD.
}

we now assume a primary mass of $1.4 M_{\odot}$ and a total secondary mass at the time of explosion of $0.5 M_{\odot}$, we obtain for a range of core masses:

$$
\begin{array}{lll}
P_{\text {orb }}\left(0.2 M_{\odot}\right)=6.74 \mathrm{~d} & \Rightarrow & V_{\text {orb }}=103 \mathrm{~km} \mathrm{~s}^{-1} \\
P_{\text {orb }}\left(0.25 M_{\odot}\right)=23.7 \mathrm{~d} & \Rightarrow & V_{\text {orb }}=68 \mathrm{~km} \mathrm{~s}^{-1} \\
P_{\text {orb }}\left(0.3 M_{\odot}\right)=69.4 \mathrm{~d} & \Rightarrow & V_{\text {orb }}=47 \mathrm{~km} \mathrm{~s}^{-1} \\
P_{\text {orb }}\left(0.35 M_{\odot}\right)=171.8 \mathrm{~d} & \Rightarrow & V_{\text {orb }}=35 \mathrm{~km} \mathrm{~s}^{-1}
\end{array}
$$

where $V_{\text {orb }}$ is the total orbital velocity. Using the same component masses, we record for comparison that $P_{\text {orb }}=1 \mathrm{~d}$ corresponds to $V_{\text {orb }} \approx 194 \mathrm{~km} \mathrm{~s}^{-1}$ and $P_{\text {orb }}=1 \mathrm{~h}$ to $V_{\text {orb }} \approx$ $560 \mathrm{~km} \mathrm{~s}^{-1}$. The orbital velocities at explosion in the simulations of Han \& Podsiadlowski (2004) range from $80-230 \mathrm{~km} \mathrm{~s}^{-1}$ for MS and subgiant donors.

RS Ophiuchi has an orbital period of $\approx 457 \mathrm{~d}$. Inverting Eq. (2) above, this corresponds to a core mass for the donor of slightly over $0.4 M_{\odot}$, still within the mass range for a LMWD should the envelope be removed.

The arguments in Sect. 3.2 suggest that LMWDs are produced by giant donors, as long as the orbital period is not so long that the core has already grown to $0.5 M_{\odot}$ by the time their tenuous envelopes are stripped by the supernovae ejecta. So, in contrast to the high-velocity WDs observed by Oppenheimer et al. (2001) and interpreted by Hansen (2003) as remnants of SNe Ia with main-sequence donors, it would be consistent to find that the single LMWD population was not significantly kinematically heated.

In Sect. 3.2 we identified two potential SN Ia formation channels able to produce LMWDs. We note that whilst one of those sets of donor stars (red giants) would leave LMWD remnants with low runaway velocities, the other (hot subdwarfs) would result in high-velocity LMWDs.

\section{Single UCWDs as LMWDs and SN la remnants?}

We have argued that single LMWDs can be produced from single-degenerate SNe Ia with red-giant donors. Single LMWDs are inferred to exist and the most natural explanation, especially for the lower-mass LMWDs, seems to be that some singledegenerate SNe Ia occur with red giant donors. However, there is no obvious collected sample of LMWDs to examine as potential SN Ia remnants. Independently of our arguments above, it may well be that the known set of apparently single UCWDs constitutes or contains a useful sample of single LMWDs. When a suitable sample of single LMWDs becomes available our work should be extended.

\subsection{The UCWD sample}

In selecting sub-samples of the WD population, UCWDs (see, e.g. Harris et al. 1999 \& 2001; Gates et al. 2004; Wolf 2005) are clear outliers in a colour-colour diagram (see Fig. 1). Their optical colours distinctly separate them from the normal WD population, and they are an interesting curiosity in appearing to become bluer as they cool down, possibly due to the effects of collisionally induced absorption (CIA) by hydrogen molecules in the atmosphere (Bergeron et al. 1994; see also Kowalski \& Saumon 2006). When CIA affects only the infrared part of the spectrum, WDs are classed as cool; if CIA also affects the optical colours, then the WD is admitted into the select group of UCWDs (see Fig. 1; also Wolf 2005). The transition temperature between cool and ultra-cool is $\lesssim 4000 \mathrm{~K}$. 


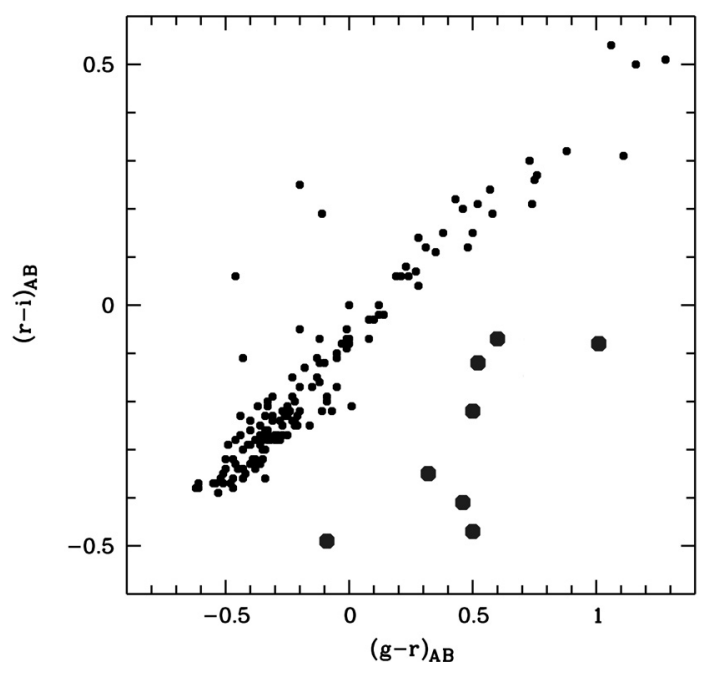

Fig. 1. A colour-colour diagram of white dwarfs. The known ultra-cool white dwarfs are represented by large circles, while the Kleinman et al. (2004) sample of normal WDs are shown as small circles. Table 1 lists the UCWDs plotted here along with the relevant references. (Adapted from Wolf (2005).)

The known UCWDs constitute a clean observational sample; they are easy to identify, and with such low luminosities (notably in the ultraviolet and near infrared) and line-free spectra, it would be hard to hide a light-emitting close companion that is anything but another UCWD (see Sect. 4.3). Table 1 contains the UCWD sample we use. The estimates of the tangential velocities for these objects depend on their assumed distances, and Table 1 shows the range of velocities obtained for assumptions taken from the literature. For that sample we shall adopt the tangential velocities obtained by taking the absolute magnitude of LHS 3250 (which is the only UCWD with a known parallax) to be representative of the whole sample.

Since we first began this work, a new sample of twenty-four UCWD candidates has been presented by Vidrih et al. (2007). We are not convinced that they are cold enough to conform to our strict criteria as UCWDs, which may mean that this sample is more likely to be contaminated by non-LMWD objects (see Sect. 4.2). However, in our later figures we shall show for comparison this new, independent, sample alongside the smaller set from Table $1^{8}$.

\subsection{Are the observed UCWDs mostly LMWDs?}

The one UCWD with a known parallax (LHS 3250) has an absolute magnitude of $M_{\mathrm{V}}=15.72$. This is brighter than expected for anything other than an LMWD; hence Bergeron \& Leggett (2002) conclude that the mass of LHS 3250 is $0.23 M_{\odot}{ }^{9}$. Unfortunately we do not have such good mass estimates for all single UCWDs.

The argument that the observed UCWDs are mostly LMWDs is partly built upon theoretical WD cooling tracks. Whereas a $0.6 M_{\odot}$ WD takes more than 9 Gyr to cool to $4000 \mathrm{~K}$

\footnotetext{
8 We have not included an extremely recent sample of seven very cool white dwarfs (Harris et al. 2008).

${ }^{9}$ Harris et al. (2001) also suggest that LHS 3250 is probably a lowmass helium core WD based on its absolute magnitude; however, the earlier paper by Harris et al. (1999) noted that a pair of UCWDs in a binary might help explain the higher luminosity without recourse to a low-mass WD.
}

(after the formation of the WD), the $0.3 M_{\odot}$ WD of equivalent composition takes less than 4 Gyr (Bergeron et al. 2001; Bergeron et al. 2005). As the cooling of WDs is a function of composition it is likely that not all UCWDs are LMWDs (see, e.g. Hansen 1999 , who requires WD masses $\lesssim 0.25 M_{\odot}$ in order for those objects to cool to $4000 \mathrm{~K}$ within $7 \mathrm{Gyr})^{10}$. Extremely low-mass WDs $\left(\lesssim 0.17 M_{\odot}\right)$ seem to cool more slowly than more massive WDs, due to the retention of a relatively thick hydrogen envelope (e.g. Panei et al. 2007). It is not clear whether the LMWD remnants that have been formed by having their envelopes forcibly removed by a supernova shockwave will retain a thick hydrogen envelope.

Hence we expect that the observed, apparently single, UCWDs are dominated by single LMWDs if they exist, partly as they are significantly more luminous than massive WDs and hence more likely to be discovered. Although it is unfortunate that we cannot prove what fraction of single UCWDs are LMWDs, in Sect. 4.4 we show that the observed numbers of single UCWDs could all be single LMWDs produced via an SN Ia explosion.

\subsection{Are the apparently single UCWDs mostly single?}

Seven of the eight UCWDs in Fig. 1 have no known companion. The exception - SDSS J0947 - has a common proper motion companion (Gates et al. 2004). The 20 arcsec angular separation of J0947 from its potential companion implies a projected separation of over $2 \times 10^{5} R_{\odot}$ for a distance of $47 \mathrm{pc}$; if that companion really forms a binary with J0947 (rather than being chance projection), it could not have influenced the evolution of the progenitor of the J0947 UCWD.

We do, however, need to consider whether these apparently single UCWDs really are single. We fully expect that UCWDs should exist in binary systems, but in the following we argue that these are unlikely to contaminate our sample; in many cases, a companion would even completely hide a UCWD.

\subsubsection{Non-degenerate companions}

An M-dwarf with an absolute $V$ magnitude of $\sim 16$ - similar to the UCWD LHS 3250 - would have a mass of $\sim 0.1 M_{\odot}$ (Delfosse et al. 2000). Such a star would be bright in the infrared, where the emission of UCWDs is strongly suppressed ${ }^{11}$. Furthermore, M-dwarfs are rich in spectral lines, so could be easily detected.

\subsubsection{White-dwarf companions}

A binary containing a non-ultra-cool WD should be identifiable: an advantage of UCWDs is that their low luminosity and featureless spectrum makes it hard for them to possess an undetectable hotter WD companion. Their characteristic spectral energy distributions mean that a non-UCWD companion would be brighter than the UCWD in either near infrared or ultraviolet light. However, a spectroscopic UCWD-UCWD binary would be difficult to distinguish from a single UCWD. We have no reason

\footnotetext{
${ }_{10}$ We note in advance that this mass of $\lesssim 0.25 M_{\odot}$ is in good agreement with our results in Sect. 4.6 combined with the core-mass orbital-period relation; it is thus self-consistent.

${ }^{11}$ For example, a $\sim 0.1 M_{\odot}$ star is about six magnitudes brighter in the $J$ band than the $V$ band (Delfosse et al. 2000), wheras LHS 3250 and SDSS 1337 are dimmer in the $J$ than $V$ bands (Harris et al. 2001). This trend is greater at longer wavelengths.
} 
Table 1. Distances and tangential velocities of the UCWD sample (as in Fig. 1).

\begin{tabular}{|c|c|c|c|c|}
\hline \multirow[t]{2}{*}{ Name } & \multicolumn{2}{|c|}{ Estimated properties for $M_{\mathrm{V}}=15.7^{a}$} & \multicolumn{2}{|c|}{ Estimated properties for $M_{\mathrm{V}}=16.5 \pm 1.0^{b}$} \\
\hline & Distance (pc) & Tangential velocity $\left(\mathrm{km} \mathrm{s}^{-1}\right)$ & Distance $(\mathrm{pc})$ & Tangential velocity $\left(\mathrm{km} \mathrm{s}^{-1}\right)$ \\
\hline LHS $3250^{(1)}$ & 30 & 81 & \multicolumn{2}{|c|}{ LHS 3250 has a known parallax } \\
\hline LHS $1402^{(2)}$ & $24^{c}$ & 56 & $12-31^{d}$ & $28-72$ \\
\hline SDSS J0947 (3) & 47 & 18 & $21-52$ & $8-21$ \\
\hline SDSS J1001 ${ }^{(3)}$ & 64 & 107 & $28-71$ & $47-119$ \\
\hline SDSS J1220 (3) & 64 & 154 & $28-71$ & $68-170$ \\
\hline SDSS J1337 $7^{(4)}$ & 54 & 46 & $23-58$ & $20-49$ \\
\hline SDSS J1403 $3^{(3)}$ & 44 & 60 & $19-49$ & $26-66$ \\
\hline COMBO-17 J1143(5) & 169 & 42 & $37-188$ & $9-46$ \\
\hline
\end{tabular}

For all objects except LHS 3250, the distance is inferred from an assumed absolute magnitude. ${ }^{a}$ I.e. taking LHS 3250, with a known trigonometric parallax, as representative of all UCWDs; ${ }^{b}$ i.e. using a conservative assumption adopted by Salim et al. (2004), Gates et al. (2004) and Wolf (2005); ${ }^{c}$ for LHS 1402, we compare a $B$ magnitude of 18.32 (Oppenheimer et al. 2001) with LHS 3250 (18.85; Harris et al. 1999); ${ }^{d}$ correcting for different photometry, we compare a $B$ magnitude of 18.2 with the $g$ magnitude (20.04) of SDSS J1001.

References. ${ }^{(1)}$ Harris et al. (1999); (2) Oppenheimer et al. (2001); ${ }^{(3)}$ Gates et al. (2004); (4) Harris et al. (2001); (5) Wolf (2005).

for thinking that such binary UCWDs do not exist, and Harris et al. (2008) have discovered one system which might eventually be expected to become such a binary. Although we do not expect that they are common enough to dominate the population, the possibility that the apparently single UCWDs have extremely cool or faint WD companions should be studied further.

\subsubsection{Neutron-star companions}

From the arguments in Sect. 2.1 it is clear that, in order to make a LMWD in a system where the NS was produced in a corecollapse supernova, the LMWD progenitor must lose mass after the formation of the NS. Hence, either mass transfer onto the NS or a common-envelope phase (Paczyński 1976) would be required. In the former case, the pulsar is expected to be recycled into a millisecond pulsar (MSP).

It is thus reasonable to expect that UCWDs might be seen as companions to millisecond pulsars. This has been claimed for PSR J0751+1807 (Bassa et al. 2006a), although the LMWD in that system is not quite formally ultra-cool. The nearby millisecond pulsar J0437-4715 also seems to be accompanied by a borderline UCWD (Danziger et al. 1993). It could be argued that, since we do see LMWDs with pulsars, it is not a surprise that some of these LMWDs happen to be in systems where we do not see the pulsar (see also van Kerkwijk et al. 1996; van Kerkwijk et al. 2000; Bassa et al. 2006b). However, the space densities of these different samples are extremely different. The companions to pulsars have been discovered because they are orbiting a pulsar, unlike the photometrically-discovered UCWD sample: Bassa, van Kerkwijk \& Kulkarni state that PSR J0751+1807 is $\sim 0.6 \mathrm{kpc}$ away, considerably further than any of our UCWD sample (see Table 1), and PSR J0437-4715 is 150 pc away (Danziger et al. 1993), beyond all but one of the photometricallydiscovered UCWDs.

A crude comparison between the possible detection volumes of the single UCWDs and the pulsar companions gives $\sim(600 / 70)^{3} \approx 630$. Here we have considered that COMBO-17 $\mathrm{J} 1143$, as a serendipitously discovered object, does not give a good measure of the systematic detection volume of the UCWDs in our sample. This suggests that, even if the companions to PSR J0751+1807 and PSR J0437-4715 were cool enough to be UCWDs, the seven systematically discovered UCWDs in our sample are $\sim 2200$ times more abundant than those with pulsar companions. This factor can be increased by another order of magnitude if one assumes that all the known nearby pulsars have been studied, but that only a tenth of the sky has been surveyed to the same depth as the field that produced the SDSS UCWD sample. To only observe one pulsar in 20 000 supposedly MSPcontaining objects would imply extremely narrow-beam pulsar emission. Unless those unseen MSPs were somehow unusual, this would suggest that many more pulsars exist than we currently expect, significantly worsening any mismatch between the inferred birthrates of LMXBs and MSPs (e.g. Kulkarni \& Narayan 1988; Lorimer 1995; Pfahl, et al. 2003) $)^{12}$.

If a NS was formed in the system through AIC and was not subsequently spun up, it could reasonably be expected not to emit pulsar radiation. A black-hole companion would, of course, not be expected to emit pulsar radiation, but a local space density for such black-hole binaries of $\sim 10^{-5} \mathrm{pc}^{-3}$ (see Sect. 4.4) is highly unexpected (see, e.g. Romani 1998).

\subsection{UCWD population numbers}

Gates et al. (2004) estimated a space density for UCWDs of $3 \times$ $10^{-5} \mathrm{pc}^{-3}$ from a sample of 6 objects found in the Sloan digital sky survey ${ }^{13}$. This rough figure does compare with an estimate of the SN Ia rate integrated over time and space. The local stellar density of $0.1 M_{\odot} \mathrm{pc}^{-3}$ (Binney \& Merrifield 1998), combined with a mass for the thin disc of $\sim 4 \times 10^{10} M_{\odot}$ and the assumption that the mass fraction of UCWDs is constant throughout the disc, produces an estimate for the number of UCWDs of $\sim 10^{7}$.

We approximate the current SN Ia rate in the Galactic disc as $\sim 7 \times 10^{-3} / \mathrm{yr}$ (using the same disc mass as above and the SN Ia rate per unit mass of Mannucci et al. 2005). Multiplying this rate by a Galactic age of $\sim 10$ Gyr leads to an estimate of a total of $\sim 7 \times 10^{7}$ remnants $^{14}$.

12 If we repeat this estimate for the new Vidrih et al. sample of 24 UCWDs, which extends to a distance of $180 \mathrm{pc}$ but over only 250 square degrees of the sky, then we have $\sim(600 / 180)^{3} \times(24 / 2) \times$ (41523/250), i.e. single UCWDs outnumber those with MSPs by a factor of over 70000 (over 27000 if UCWDs are twice as bright as Vidrih et al. assume and their survey depth extends to $250 \mathrm{pc}$ ).

13 For the Viridh et al. sample, we estimate a space density of $\sim 16 \times$ $10^{-5} \mathrm{pc}^{-3}$ for a survey depth of $180 \mathrm{pc}$, and $\sim 6 \times 10^{-5} \mathrm{pc}^{-3}$ for a survey depth of $250 \mathrm{pc}$.

14 The supernova rate may well have been different in the past. Hansen (2003) made estimates for the number of SN Ia remnants in the Milky Way based on the amount of iron in the Galaxy and found a range between $4 \times 10^{7}$ and $2.2 \times 10^{8}$ objects. 
Even if we halve this number of remnants to allow for the WD cooling time (see Sect. 4.2), the number of single UCWDs is easily consistent with them being produced through SN Ia. Indeed, these numbers suggest that only a subset of SNe Ia produces single UCWDs, which is as expected if only a subset of the SN Ia formation channels can produce LMWDs (see Sects. 3.1 and 3.2).

\subsection{Population kinematics: method}

The lack of lines in UCWD spectra means that we do not know the radial velocities for our sample. However, we can examine the population kinematics using only the information from the tangential velocities. We now investigate whether their observed space velocities are consistent with single LMWDs released from binaries with a range of orbital periods.

For a range of inital parameters, we integrated the motion of $10^{5}$ assumed SN Ia remnants for up to $10 \mathrm{Gyr}$ through the Galactic potential (using a similar procedure to Brandt \& Podsiadlowski 1995), orientating the orbital velocity vector of the donor at random when the binary is disrupted. For each integrated population, we used a single value of orbital period (and hence orbital velocity) at the time of the explosion ${ }^{15}$. For calculating the orbital velocity at a given orbital period, donor stars are assumed to be $0.5 M_{\odot}$ at the time of the explosion, and the WDs are assumed to explode at a mass of $1.4 M_{\odot}$. Equation (1) shows that our results should be relatively insensitive to those assumptions, but in the future we intend to perform this procedure using the output of our binary population synthesis calculations.

Each remnant is initially located at random within an axisymmetric Galaxy modelled by two exponential scale-heights (vertical and radial). The axisymmetry is also exploited for computational efficiency: at each integration time-step the view from Earth is calculated at all points on the solar circle. A further assumption is that the remnants can be observed to a distance of $160 \mathrm{pc}$ - broadly appropriate for UCWDs. Within such a small volume, the space velocities should only be a very weak function of distance.

The Galactic potential was taken from Paczyński (1990), using the parameters in Brandt \& Podsiadlowski (1995), with the addition of scattering from giant molecular clouds (GMCs) randomly distibuted within the Galactic disc. The total mass in these GMCs is assumed to be $1.2 \times 10^{9} M_{\odot}$, with a mass spectrum exponent such that $\mathrm{d} N / \mathrm{d} \log m \propto m^{-1.7}$ (see, e.g. Digel et al. 1996; Binney \& Merrifield 1998).

We do not include any kick imparted by the supernova ejecta in our simulations, since the simulation of Marietta et al. (2000) show that the kicks due to the supernova interaction $\left(86 \mathrm{~km} \mathrm{~s}^{-1}\right.$ and $49 \mathrm{~km} \mathrm{~s}^{-1}$ for their main-sequence and subgiant donors, respectively, with no kick given to the core of the giant donor) are generally small compared the orbital-velocity kick from the break-up of the binary.

Since the initial disc scale-height of the progenitor population is uncertain, we present our results for a range of values. The vertical scale-height of massive stars is 75 pc (van der Kruit 1987), and $200 \mathrm{pc}$ is an approximation to a more generic thin disc population (e.g. Ojha et al. 1996, who find a scaleheight of $260 \pm 50 \mathrm{pc}$ for the Galactic thin disc and $760 \pm 50$ for the thick disc; see also Kroupa et al. 1993). Given previous speculations

\footnotetext{
15 We expect that in reality SN Ia explosions will occur over a range of orbital periods, but the current data only allow us to investigate broad trends.
}

that UCWDs are so cool because they are very old objects, we also modelled initial scale-heights of $500 \mathrm{pc}, 1 \mathrm{kpc}$ and $4 \mathrm{kpc}^{16}$.

\subsection{Population kinematics: Results}

Figures 2 and 3 present the results of our integrations for orbital periods at the time of the supernova of 1, 10 and 100 days, as well as a population which received no kick. The population which received no kick seems to be difficult to reconcile with the observed tangential velocities, except for the most extreme range of luminosities consistent with the literature combined with an inital vertical scale height of $4 \mathrm{kpc}$ (or greater). The population released from a one day orbital period also appears inconsistent with the data, whichever initial scale-height is assumed. These conclusions are supported by applying the Kolmogorov-Smirnov test to compare our simulations with the tangential velocity distribution produced by assuming LHS 3250 is a typical UCWD (Table 1). Each plot contains a Kolmogorov-Smirnov acceptance probability $\left(P_{x}\right)$ for each curve ${ }^{17}$.

Given the considerable uncertainties, we consider that the extremely favourable Kolmogorov-Smirnov test for the population released from 100 day orbits with a $4 \mathrm{kpc}$ scale height should not be over-interpreted, especially as a real SN Ia population would be expected to have a range of orbital periods. Both the uncertainty in the distances to UCWDs and in the formation kinematics restricts our ability to draw quantitative conclusions. However, both the observational samples presented in Figs. 2 and 3, with their different assumptions, seem to suggest that these apparently single LMWDs have experienced some kick.

The broadly favoured period range at explosion is between 10 and 100 days, with the longer orbital periods preferred for a larger initial scale-height. The sample presented by Vidrih et al. leads to a lower set of tangential velocities, and their sample strongly prefers 100 over $10 \mathrm{~d}$. This may be because they assume fainter absolute magnitudes than we adopt, and hence systematically produce lower velocities than us. Alternatively, their larger but less cool sample may be more likely to be contaminated by objects which are not single LMWDs.

This period range of 10-100 d approximately encompasses the core masses appropriate for the production of LMWDs, exactly as might be expected if the stellar evolution was truncated by the loss of a giant star's envelope. This is consistent with an explanation of these UCWDs as being descended from giant donors. We note, however, that we cannot exclude a thick disc origin for these objects with an arbitrarily small kick. Although we cannot use this apparent kick as a definitive signature of an origin in type Ia supernovae, a thick disc origin would not falsify our SN Ia hypothesis, but it would suggest longer orbital periods at the point of explosion.

\section{Discussion}

The main conclusion of this study is that single LMWDs constitute indirect evidence that $\mathrm{SNe}$ Ia are formed through the single-degenerate channel, specifically from systems with redgiant donors. A field population of truly single LMWDs, especially with masses less than $\sim 0.4 M_{\odot}$, would seem to require an SN Ia origin, or a significant revision of our understanding

\footnotetext{
${ }_{16}$ Note that the collective tangential velocities of the sample in Table 1 are clearly inappropriate for objects from a halo population.

${ }^{17}$ Here $1-P_{x}$ gives the probability that the data and model $x$ are drawn from different distributions, so we can reject a model with $P=0.01$ with $99 \%$ confidence.
} 

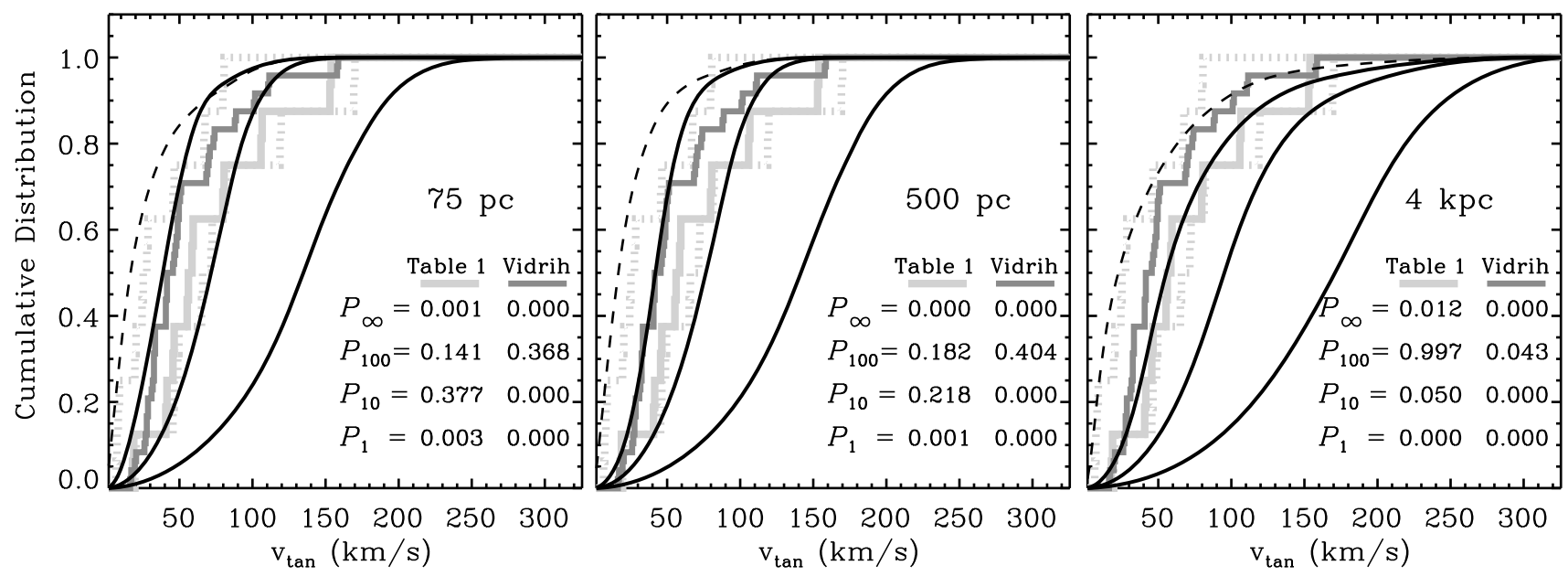

Fig. 2. Comparison of the tangential velocities resulting from our Galactic integrations (smooth black curves), with the tangential velocities of the observed UCWDs (grey step functions). The solid light grey step function uses the distance estimates from the assumption that LHS 3250 is representative of the UCWD population, and the broken grey step functions encompass the wider range of distances in Table 1 . The dark grey step function uses the new sample of UCWD candidates from Vidrih et al. (2007), adopting their assumptions for the UCWD distances. The solid black curves in each panel are for orbital periods at the time of the explosion of 100, 10 and 1 days (left to right). The dashed black curve represents a population which receives no "kick" due to the break-up of a binary system. The Kolmogorov-Smirnov acceptance probabilities for the individual models are given in each panel, compared to both the objects in Table 1 and the Vidrih et al. sample. Here $P_{\infty}$ refers to the model with no added "kick" velocity from binary break-up and $P_{100}, P_{10}$ and $P_{1}$ to the curves representing the 100, 10 and 1 day orbital-period populations, respectively (see text). The simulations assumed initial disc scale-heights of $75 \mathrm{pc}, 500 \mathrm{pc}$ and $4 \mathrm{kpc}$ (left to right). If these objects were once the cores of red-giant donor stars in SN Ia producing systems, the simplest expectation would be for orbital periods of $\sim 100 \mathrm{~d}$ (see text).
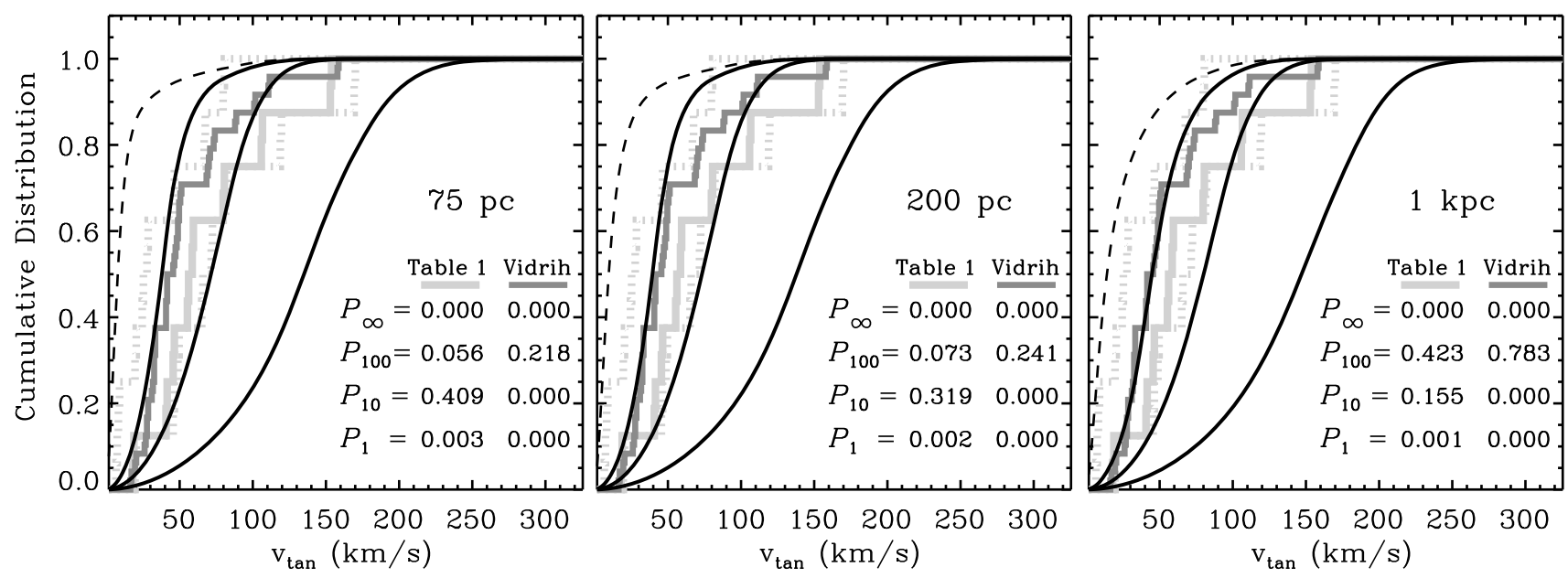

Fig. 3. As Fig. 2, for simulations that do not include any scattering from giant molecular clouds. The plots assume initial disc scale-heights of $75 \mathrm{pc}, 200 \mathrm{pc}$ and $1000 \mathrm{pc}$ (left to right).

of either $\mathrm{CV}$ evolution or $\mathrm{AIC}^{18}$. That is based on evolutionary arguments (Sects. 2 and 3) and does not rely on any kinematic information.

We have simultaneously demonstrated that there is a natural formation channel for single LMWDs which does not require any unexpected modification of single-star evolution.

Those conclusions are independent of the nature of apparently single UCWDs. However the currently observed UCWD sample may be dominated by LMWDs, and we find that the kinematics of the known single UCWDs seem to be consistent with an origin in $\mathrm{SNe}$ Ia with red-giant donor stars. For reasonably

\footnotetext{
${ }^{18}$ It may be that explosions of sub-Chandrasekhar-mass WDs occur which are not seen as SN Ia but are capable of stripping the envelopes of RG donor stars. That would provide a further possible channel for the formation of single LMWDs.
}

broad assumptions, they even suggest that the orbital periods at the time of explosion were $\lesssim 100 \mathrm{~d}$. The population of UCWDs require further study in order to strengthen those specific conclusions.

\subsection{SN la progenitor evolution}

The implication of the existence of single LMWDs is that at least some single-degenerate SNe Ia occur with the donor star on the giant branch at the time of the explosion.

Both the high-velocity WD and single LMWD populations seem to contain members originating in SN Ia explosions. Dwarf star donors at the time of explosion should acquire higher space velocities than giant donors (due to their shorter orbital periods), but the less extended donors should be less stripped by the 
ram pressure of the explosion (Marietta et al. 2000) and hence go on to produce higher-mass WDs than a giant which loses its envelope prior to helium ignition. Hence the Oppenheimer et al. (2001) sample of high-velocity WDs considered by Hansen (2003) do not need to be low-mass for an SN Ia origin to be reasonable, nor do single LMWDs need to have high space velocities to invite an SN Ia explanation for their production. LP 40022 (Kawka et al. 2006) is notable for being an extreme object using either selection criterion.

A simplistic comparison of the remnant space densities quoted for the Oppenheimer et al. (2001) high-velocity sample $\left(1.8 \times 10^{-4} \mathrm{pc}^{-3}\right)$ and the Gates et al. (2004) UCWDs $\left(\sim 3 \times 10^{-5} \mathrm{pc}^{-3}\right)$ suggests that the supersoft channel is almost an order of magnitude more important than the red-giant channel, though presumably some single LMWDs destined to be single UCWDs have not yet cooled sufficiently to become UCWDs, and some UCWDs may not be LMWDs. The relative importance of these formation channels will be an interesting quantity to constrain with future data and compare with binary population synthesis models. Perhaps more decisively, the estimates of Hansen (2003) and Sect. 4.4 imply that the total number of observed remnants is consistent with the expected number of past SNe Ia in our Galaxy. If this is confirmed by future work, it would leave room for only a minority of SNe Ia to result from double-degenerate systems.

One curiosity of our study of UCWDs is that the range of orbital periods we infer for the SN Ia progenitors at explosion is only broadly consistent with that predicted by Hachisu \& Kato (2001) for systems with red-giant donors. Their models produce no SNe Ia with final orbital periods $2.5 \mathrm{~d} \lesssim P_{\text {orb }} \lesssim 60 \mathrm{~d}$; most of the final parameter space for red-giant donors has $100 \mathrm{~d} \lesssim$ $P_{\text {orb }} \lesssim 1000 \mathrm{~d}$. Our favoured assumptions seem to indicate orbital periods on the shorter side of $100 \mathrm{~d}$, i.e. the kinematic signal seems to be a little stronger than expected. A possible resolution could be that our use of UCWDs is biased and preferentially selects systems with shorter orbital periods because they produce lower-mass WDs, which cool more rapidly. Or perhaps the progenitor population is preferentially from the thick disc, and hence the remnants falsely appear kinematically hotter than in our models. It may also be that some non-LMWD single UCWDs from the thick disc or Galactic halo could be contaminating these results. Alternatively, this may be an indication that the models need some modification; for example the systems containing red-giant donors might produce $\mathrm{SNe}$ Ia earlier than expected, or perhaps a subset of systems from the supersoft channel does not explode until their donor stars have evolved more than the current models predict.

\subsection{The nature of UCWDs}

We encourage further work on the nature of UCWDs. It is important to confirm that the apparently single UCWDs really are single. High signal-to-noise searches for any spectral features that allow radial-velocity measurements would be worthwhile. GAIA astrometry is a long-term hope for examining the single status of UCWDs, as well as providing accurate distances to all these objects.

It is also important to understand the mass distribution of the UCWD population. The inferred mass of the best-studied UCWD, LHS 3250, (0.23 $M_{\odot}$ (Bergeron \& Leggett 2002)) makes it a clear LMWD and hence a good candidate SN Ia remnant, but no other apparently single UCWD has a wellconstrained mass estimate that we are aware of.

\subsection{Outlook: a definitive signature?}

A sample of non-UCWD single LMWDs has distinct advantage over the UCWDs in that the prescence of spectral lines allows for radial velocity measurements (see, e.g. Maxted et al. 2000a). The significant difficulty for these objects is in selecting a large sample: whilst UCWDs stand out from survey photometry, hotter LMWD do not. Eisenstein et al. (2006) used the Sloan digital sky survey to produce a catalogue of over 9000 white dwarfs, and identify 13 WDs with masses $<0.3 M_{\odot}$, of which 7 have masses $\leq 0.2 M_{\odot}$. These objects should be investigated for signs of a companion NS, or for radial velocity variations.

With a substantial sample of single LMWDs, it would make sense to use directional information about the space velocities of the objects rather than just the magnitudes of the transverse velocities. We expect that the use of such information will help distinguish between a large initial scale-height, long orbital period population and a thinner, shorter-period population. Our conclusions would be considerably stronger if we were sure about the initial kinematics of these stars; it is important to try to determine whether the remnants come from the thick disc.

Once the single LMWD sample becomes large enough, it could potentially be split into sub-samples with different WD masses. If our model is correct, then less massive single LMWDs should be kinematically hotter than more massive single LMWDs (see Sect. 3.4).

Since we began this work, van Leeuwen et al. (2007) and Kilic et al. (2007a) have searched for companions to LMWDs in the radio and optical wavebands, respectively. The search by van Leeuwen et al. of LMWDs for radio pulsations found none "down to flux densities of $0.6-0.8 \mathrm{mJy} \mathrm{kpc}^{-2}$ ", and concluded that "a given low-mass helium-core white dwarf has a probability of $<0.18 \pm 0.05$ of being in a binary with a radio pulsar". For four WDs with masses $<0.2 M_{\odot}$, Kilic et al. found: "None of these white dwarfs show excess emission from a binary companion, and radial velocity searches will be necessary to constrain the nature of the unseen companions" ${ }^{\prime 19}$. Our paper suggests that the assumption that there are unseen companions is not nessecary.

\subsection{Runaway hot subdwarfs}

Perhaps the most interesting possibility for the evolutionary state of the donor in a single-degenerate SN Ia is that of a hot subdwarf ( $\mathrm{sdO}$ or sdB) star. This would naturally allow short orbital periods $(\sim 1 \mathrm{~h})$ and also naturally produce extremely lowmass WDs, as recently observed in the runaway WD LP 400-22 (Kawka et al. 2006), which is inferred to have a mass of $0.17 M_{\odot}$ and a tangential velocity of $414 \pm 43 \mathrm{~km} \mathrm{~s}^{-1}$. This would also provide a natural explanation for stars like the runaway hot subdwarf US 708 (discovered by Hirsch et al. 2005). We feel that an $\mathrm{SN}$ Ia origin for this object is more satisfying than a scenario combining dynamical ejection from the supermassive black hole in the Galactic centre with the simultaneous merger of two helium WDs (as speculated by Hirsch et al.). The orbital velocity in this case may well be augmented by a kick due to an impulse from the supernova shock (e.g. Marietta et al. 2000).

The evolution of the WD-sdB binary system KPD 1930+2752 (see, e.g. Maxted et al. 2000b) has been investigated by Ergma et al. (2001). They conclude that this system is likely to eventually result in a merger of two WDs (see also

\footnotetext{
19 A companion to one of these Kilic et al. LMWDs has been found by such a radial velocity search (Kilic et al. 2007b).
} 
Geier et al. 2007). However we see no reason why similar systems could not produce an SN Ia via a single-degenerate channel, hence producing such objects as US 708 and then LP 400-22.

\section{Summary and conclusions}

We have considered the formation of apparently single LMWDs in general, concluding that the most natural scenario for the formation of single LMWDs is that they are the remnants of donor stars in single-denenerate SNe Ia. Indeed, lone LMWDs should be expected if some single-degenerate $\mathrm{SNe}$ Ia do occur with giant donor stars, as inferred from the observations of Patat et al. (2007), notably if the donors lose a significant fraction of their envelopes, as predicted for giant donors (Marietta et al. 2000; Iben \& Tutukov 1984). The observations of Maxted et al. (2000a), van Leeuwen et al. (2007) and Kilic et al. (2007a) are all in support of the existence of a population of genuinely single LMWDs.

It seems difficult for the majority of apparently single UCWDs to posess companions, and we have adopted them as a useful sample of single LMWDs. We have integrated a population of SN Ia donor remnants through a simple Galactic potential and compared the results of those calculations to the known space velocities of apparently single UCWDs. Our results are consistent with the single low-mass UCWDs having once been red-giant donor stars at the time of an SN Ia explosion, as predicted for single LMWDs.

A unified picture emerges in which the high-velocity WDs are remnants of main-sequence donors in SNe Ia (as suggested first by Hansen, 2003), and a kinematically cooler population of single LMWDs were once giant donors in long-period SN Ia progenitors: their longer orbital periods led to a lower runaway velocity wheras their tenuous envelopes were stripped more easily by the supernova ejecta to produce LMWDs.

Furthermore, it seems plausible that runaway LMWDs such as LP 400-22 and runaway hot subdwarf stars such as US 708 originate from donor stars in short-period $(\sim 1 \mathrm{~h}) \mathrm{SN}$ Ia systems. We will explore this idea in more detail in a future paper.

Acknowledgements. We thank Uli Heber for very interesting conversations and for bringing the issue of runaway hot subdwarfs to our attention. Questions from Marten van Kerkwijk helped improve the clarity of our arguments, and we thank an anonymous referee for their useful comments. Discussions with the stellar group at Oxford were also useful.

S.J. has been supported by PPARC grant PPA/G/S/2003/00056 \& Global Jet Watch, and C.W. by a PPARC Advanced Fellowship. Z.H. visited Oxford thanks, in part, to a Royal Society UK-China Joint Project Grant (Ph.P. and Z.H.). This work was partly supported by the National Science Foundation of China under Grant Nos. 10521001, 10433030 and 2007 CB815406 (Z.H.) and a European Research \& Training Network on Type Ia Supernovae (HPRN-CT20002-00303)

\section{References}

Adams, T., Davies, M. B., \& Sills, A. 2004, MNRAS, 348, 469 Bassa, C. G., van Kerkwijk, M. H., \& Kulkarni, S. R. 2006a, A\&A, 450, 295 Bassa, C. G., van Kerkwijk, M. H., Koester, D., \& Verbunt, F. 2006b, A\&A, 456, 295

Bedin, L. R., Salaris, M., Piotto, G., et al. 2008, ApJ, 679, L29

Bergeron, P., \& Leggett, S. K. 2002, ApJ, 580, 1070

Bergeron, P., Ruiz, M.-T., S. K., Saumon, D., \& Wesemael, F. 1994, ApJ, 423, 456

Bergeron, P., Leggett, S. K., \& Ruiz, M. T. 2001, ApJS, 133, 413

Bergeron, P., Ruiz, M. T., Hamuy, M., et al. 2005, ApJ, 625, 838

Binney J., \& Merrifield, M. 1998, Galactic Astronomy, Princeton

Brandt, N., \& Podsiadlowski, Ph. 1995, MNRAS, 274, 461
Canal, R., Ruiz-Lapuente, P., \& Burkert, A. 1996, ApJ, 456, L101 Cannizzo, J. K., Ghosh, P., \& Wheeler, J. C. 1982, ApJ, 260, L83 Danziger, I. J., Baade, D., \& Della Valle, M. 1993, A\&A, 276, 382 Davies, M. B., Ritter, H., \& King, A. 2002, MNRAS, 335, 369 Delfosse, X., Forveille, T., Ségransan, D., et al. 2000, A\&A, 364, 217 Digel, S. W., Lyder, D. A., Philbrick, A. J., Puche D., \& Thaddeus, P. 1996, ApJ, 458,561

Dubus, G., Campbell, R., Kern, B., Taam, R. E., \& Spruit, H. C. 2004, MNRAS, 349,869

Eggleton, P. P. 1971, MNRAS, 151, 351

Eisenstein, D. J., Liebert, J., Harris, H. C., et al. 2006, ApJS, 167, 40

Ergma, E., Fedorova, A. V., \& Yungelson, L. R. 2001, A\&A, 376, L9

Fink, M., Hillebrandt, W., \& Röpke, F. K. 2007, A\&A, 476, 1133

Gates, E., Gyuk, G., Harris, H. C., et al. 2004, ApJ, 612, L129

Geier, S., Nesslinger, S., Heber, U., et al. 2007, A\&A, 464, 299

Hachisu, I., \& Kato, M. 2001, ApJ, 558, 323

Hachisu, I., Kato, M., \& Nomoto, K. 1996, ApJ, 470, L97

Hachisu, I., Kato, M., \& Nomoto, K. 1999, ApJ, 522, 487

Han, Z. 2008, ApJ, 677, L109

Han, Z., \& Podsiadlowski, Ph. 2004, MNRAS, 350, 1301

Han, Z., Podsiadlowski, Ph., \& Eggleton, P. P. 1994, MNRAS, 270, 121

Han, Z., Podsiadlowski, Ph., \& Eggleton, P. P. 1995, MNRAS, 272, 800

Han, Z., Podsiadlowski, Ph., Maxted, P. F. L., Marsh, T. R., \& Ivanova, N. 2002, MNRAS, 336, 449

Hansen, B. M. S. 1999, ApJ, 520, 680

Hansen, B. M. S. 2003, ApJ, 582, 915

Hansen, B., \& Liebert, J. 2003, ARA\&A, 41, 465

Harris, H. C., Dahn, C. C., Vrba, F. J., et al. 1999, ApJ, 524, 1000

Harris, H. C., Hansen, B. M. S., Liebert, J., et al. 2001, ApJ, 549, L109

Harris, H. C., Gates, E., Gyuk, G., et al. 2008, ApJ, 679, 697

Hirsch, H. A., Heber, U., O’Toole, S. J., \& Bresolin, F. 2005, A\&A, 444, L61

Iben, I., \& Tutokov, A. V. 1984, ApJS, 54, 335

Kalirai, J. S., Bergeron, P., Hansen, B. M. S., et al. 2007, ApJ, 671, 748

Kawka, A., Vennes, S., Oswalt, T. D., Smith, J. A., \& Silvestri, N. M. 2006, ApJ, 643, L123

Kilic, M., Prieto, C. A., Brown, W. R., \& Koester, D. 2007a, ApJ, 660, 1451

Kilic, M., Brown, W. R., Allende Prieto, C., Pinsonneault, M. H., \& Kenyon, S. J. 2007b, ApJ, 664, 1088

Kilic, M., Stanek, K. Z., \& Pinsonneault, M. H. 2007c, ApJ, 671, 761

King, A. R., Rolfe, D. J., \& Schenker, K. 2003, MNRAS, 341, L35

Kleinman, S. J., Harris, H. C., Eisenstein, D. J., et al. 2004, ApJ, 607, 426

Kowalski, P. M., \& Saumon, D. 2006, ApJ, 651, L137

Kroupa, P., Tout, C. A., \& Gilmore, G. 1993, MNRAS, 62, 545

Kulkarni, S. R., \& Narayan, R. 1988, ApJ, 335, 755

Langer, N., Deutschmann, A., Wellstein, S., \& Höflich, P. 2000, A\&A, 362, 1046 Levan, A. J., Wynn, G. A., Chapman, R., et al. 2006, MNRAS, 368, L1

Li, X.-D., \& van den Heuvel, E. P. J. 1997, A\&A, 322, L9

Liebert, J., Bergeron, P., Eisenstein, D., et al. 2004, ApJ, 606, L147

Lorimer, D. R. 1995, MNRAS, 274, 300

Mannucci, F., Della Valle, M., Panagia, N., et al. 2005, A\&A, 433, 807

Marsh, T. R., Dhillon, V. S., \& Duck, S. R. 1995, MNRAS, 275, 828

Martin, R. G., Tout, C. A., \& Lesaffre, P. 2006, MNRAS, 373, 263

Marietta, E., Burrows, A., \& Fryxell, B. 2000, ApJS, 128, 615

Matteucci, F., \& Recchi, S. 2001, ApJ, 558, 351

Maxted, P. F. L., Marsh, T. R., \& Moran, C. K. J. 2000a, MNRAS, 319, 305

Maxted, P. F. L., Marsh, T. R., \& North, R. C. 2000b, MNRAS, 317, L41

Meng, X., Chen, X., \& Han, Zh. 2008, A\&A, 487, 625

Muno, M. P., \& Mauerhan, J. 2006, ApJ, 648, L135

Mürset, U., \& Schmid, H. M. 1999, A\&AS, 137, 473

Nomoto, K. 1982, 253, 798

Nomoto, K., \& Iben, I. 1985, ApJ, 297, 531

Nomoto, K., \& Kondo, Y. 1991, ApJ, 367, L19

Nomoto, K., Thielemann, F., \& Yokoi, K. 1984, ApJ, 286, 644

Ojha, D. K., Bienaymé, O., \& Robin, A. C. 1996, A\&A, 311, 456

Oppenheimer, B. R., Hambly, N. C., Digby, A. P., Hodgkin, S. T., \& Saumon, D. 2001, Science, 292, 698

Paczyński, B. 1976, in Structure and Evolution of Close Binary Systems, ed. P. P.

Eggleton, S. Mitton, \& J. Whelan (Dordrecht: Reidel), IAU Symp., 73, 75 Paczyński, B. 1990, ApJ, 348, 485

Paczyński, B., \& Żytkow, A. N. 1978, ApJ, 222, 604

Panei, J. A., Althaus, L. G., Chen, X., \& Han, Z. 2007, MNRAS, 382, 779

Patat, F., Chandra, P., Chevalier, R., et al. 2007, Science, 317, 924

Perlmutter, S., Aldering, G., Goldhaber, G., et al. 1999, ApJ, 517, 565

Pfahl, E., Rappaport, S., \& Podsiadlowski, P. 2003, ApJ, 597, 1036

Podsiadlowski, Ph. 2003 [arXiv: astro-ph/0303660]

Pols, O. R., Tout, C. A., Eggleton, P. P., \& Han, Z. 1995, MNRAS, 274, 964

Pols, O. R., Schröder, K.-P., Hurley, J. R., Tout, C. A., \& Eggleton, P. P. 1998,

MNRAS, 298, 525 
Rappaport, S., Di Stefano, R., \& Smith, J. D. 1994, ApJ, 426, 692

Rappaport, S., Podsiadlowski, Ph., Joss, P. C., Di Stefano, R., \& Han, Z. 1995, MNRAS, 273, 731

Riess, A. G., Filippenko, A. V., Challis, P., et al. 1998, ApJ, 116, 1009

Riess, A. G., Strolger, L.-G., Tonry, J., et al. 2004, ApJ, 607, 665

Romani, R. W. 1998, A\&A, 333, 583

Ruiz-Lapuente, P. 1997, Science, 276, 1813

Ruiz-Lapuente, P., Comeron, F., Méndez, J., et al. 2004, Nature, 431, 1069

Salim, S., Rich, R. M., Hansen, B. M., et al. 2004, ApJ, 601, 1075

Sokoloski, J. L., Luna, G. J. M., Mukai, K., \& Kenyon, S. J. 2006, Nature, 442, 276

Spruit, H. C., \& Taam, R. E. 2001, ApJ, 548, 900

Spruit, H. C., \& Taam, R. E. 2003, in From X-ray binaries to Gamma-Ray Bursts, ed. E. P. J. van den Heuvel, L. Kaper, E. Rol, \& R. A. M. J. Wijers, ASP Conf. Proc., San Francisco, 308, 323

Taam, R. E., \& Spruit, H. C. 2001, ApJ, 561, 329

van den Heuvel, E. P. J., Bhattacharya, D., Nomoto, K., \& Rappaport, S. 1992, A\&A, 262, 97 vander Kruit, P. C. 1987, in The Galaxy, Series C: Mathematical and Physical Sciences, ed. G. Gilmore, \& B. Carswell (Dordrecht: Reidel), 207, 27

Van Kerkwijk, M. H., Bergeron, P., \& Kulkarni, S. R. 1996, ApJ, 467, L89

Van Kerkwijk, M. H., Bell, J. F., Kaspi, V. M., \& Kulkarni S. R. 2000, ApJ, 530, L37

Van Leeuwen, J., Ferdman, R. D., Meyer, S., \& Stairs, I. 2007, MNRAS, 374, 1437

van Loon, J. T., Boyer, M. L., \& McDonald, I. 2008, ApJL, 680, L49

Vidrih, S., Bramich, D. M., Hewett, P. C., et al. 2007, MNRAS, 382, 515

Webbink, R. F. 1984, ApJ, 277, 355

Weidemann, V. 2000, A\&A, 363, 647

Whelan, J., \& Iben, I. 1973, ApJ, 186, 1007

Wolf, C. 2005, A\&A, 444, L49

Woosley, S. E., \& Weaver, T. A. 1994, ApJ, 423, 371

Yoon, S.-C., Podsiadlowski, Ph., \& Rosswog, S. 2007, MNRAS, 380, 933

Zamanov, R. K., Bode, M. F., Melo, C. H. F., et al. 2007, MNRAS, 380, 1053 